\title{
Experimental and Numerical Study of Anisotropic Thermal Conductivity of Magnetically Aligned PDMS/Ni Particle Composites
}

\author{
Junwei Su, Xiao Liu, Majid Charmchi, Hongwei Sun \\ Department of mechanical engineering, University of Massachusetts Lowell
}

\begin{abstract}
In this research, Polydimethylsiloxane (PDMS)/Nickel (Ni) composites with embedded $\mathrm{Ni}$ spherical particle columns were studied for thermal conductivity enhancement. The volume fraction of $\mathrm{Ni}$ particles ranged from 2 to $20 \%$ while the strength of the applied magnetic field was fixed at 0.45 Tesla. The distribution and morphology of the column structures were quantitatively analyzed using optical microscope, scanning electron microscope (SEM) and digital image processing. A reusable $3 \omega$ measurement technique was applied to measure the effective thermal conductivity of PDMS/Ni composites in the parallel direction to the magnetic field. The measured thermal conductivity was compared with the prediction from a finite element model built on the observed microscopic structures. Under a static magnetic field, $\mathrm{Ni}$ particles align parallel to the field forming columns. The results illustrated that the diameter of $\mathrm{Ni}$ columns increased with increasing particle volume fraction while the center to center spacing between columns did not change substantially under the fixed magnetic strength. The magnetically aligned particle columns significantly enhanced the thermal conductivity of PDMS compared to the randomly distributed particles by about two fold. However, the point contacts between magnetically aligned spherical fillers are the major limiting factor for the further improvement of thermal conductivity.
\end{abstract}

Keywords: Polymer-matrix composites (PMCs); Thermal conductivity; $3 \omega$ method; Finite element modeling; Microstructural analysis

\section{NOMENCLATURE}

$x \quad$ direction parallel to the film plane (in-plane)

$y \quad$ direction perpendicular to the film plane (cross-plane); parallel to the magnetic field

b heater's half width

$k \quad$ thermal conductivity

$\omega \quad$ angular modulation frequency

$p / l \quad$ the amplitude of the power per unit length of the stripe generated at a frequency of $2 \omega$

$A_{1} \quad$ a constant for boundary condition

$q^{-1} \quad$ thermal penetration depth 


\begin{tabular}{|c|c|}
\hline$\Delta T$ & temperature oscillation amplitude \\
\hline$\Delta T_{x}$ & the real part of temperature oscillation \\
\hline$\Delta T_{t}$ & theoretical temperature oscillation amplitude \\
\hline$v_{3 \omega}$ & $3 \omega$ voltage component over metal stripe \\
\hline$V$ & voltage with frequency of $1 \omega$ over the metal stripe \\
\hline$V_{3 \omega}$ & $3 \omega$ voltage component over Wheatstone bridge (Fig. 1) \\
\hline$L$ & length of unit cell \\
\hline$H$ & height of unit cell \\
\hline$R_{o}$ & resistance of metal strip \\
\hline$\lambda$ & wavenumber \\
\hline$\alpha$ & thermal diffusivity \\
\hline$\phi$ & volume fraction of nickel fillers \\
\hline$k_{p}$ & thermal conductivity of particle fillers \\
\hline$k_{m}$ & thermal conductivity of matrix (PDMS) \\
\hline$k_{e f f}$ & effective thermal conductivity of composite \\
\hline
\end{tabular}

\section{Introduction}

Low interfacial free energy, flexibility, optical transparency, chemical inertness and durability have made Polydimethylsiloxane (PDMS) a primary structural material for bioMEMS [1, 2], microfluidic [3], and other biomedical devices [4]. However, its low thermal conductivity has severely restricted the performance of some microfluidic devices such as electrokinetically driven microfluidic chips (Joule heating) [5]. Also, the use of PDMS is limited in many other potential applications such as flexible electronics, where PDMS is a promising candidate material but the lack of efficient heat transfer between the local heat source (such as embedded electronic components) and surrounding environment is still a challenging issue. Due to the non-uniform distribution of heat spots, local heat generation would increase the chip junction maximum temperature, which may cause failure of the embedded electronic devices $[6,7]$.

Enhancing the thermal conductivity of materials through embedding highly conductive fillers has been extensively studied in the past decades [8-12]. Particulate fillers have been widely utilized in sufficient quantities in polymers in thermal interface materials (TIM) for thermal management of engineering systems, such as electronic cooling. Metal particles (e.g, gold and silver) and nonmetallic fillers like ceramic powders (e.g., nanoalumina), nanoclays, carbon nanotubes, and fullerenes have been commonly applied [13-16]. Most of these particles have relatively high thermal conductivities to provide the required thermal conductance. In some 
thermal interface materials, however, there could be poor thermal transport because of the lack of continuous pathways for heat flow due to the interfaces between discrete particles (the fillers) and the polymer and lack of percolation/fractal cluster. Some synthesis processes have been developed to transform a pure PDMS material into a conductive composites material by adding carbon black powder [17] or alumina $\left(\mathrm{Al}_{2} \mathrm{O}_{3}\right)$ nanoparticles [18] into PDMS. However, the improvement in thermal conductivity was very limited despite a high volume fraction of conductive fillers which had been used in these well-dispersed PDMS composites. As the Brownian motion effect $[19,20]$ is eliminated in solid composites, compared to dispersed particles in liquids (nanofluids), the remaining parameters affecting the thermal properties of composites are the volume fraction, aspect ratio, alignment of fillers, and the thermal property of the interface. It is observed that the better the percolation filler network was constructed then, the higher thermal conductivity enhancement resulted. Indeed, the heat conduction along the filler network holds the key to the thermal conductivity enhancement [21].

Magnetorhelogical (MR) elastomers (magnetic filler embedded elastomers) relies on the microstructures formed by magnetic fillers responding to an external magnetic field to alter the mechanical stiffness of the elastomer and are very attractive for various applications ranging from automotive, aerospace to civil engineering materials [22, 23]. Moreover, these structures can be formed during the crosslinking process of elastomer, such as PDMS, to form the uniformly distributed percolation network for better thermal conduction improvement. The electrical conductivity enhancement of MR elastomers has been demonstrated in many previous works [24-28]. Very recently, Boudenne et al. [29] measured the improved thermal properties of silicone-Ni composites under a magnetic field using a heat guarded plate (HGP) method. However, there still is a lack of comprehensive research on the filler structures in the polymer matrix and the related thermal properties of MR elastomers.

The $3 \omega$ method, developed by Cahill [30], is an effective technique to measure the thermal conductivity of a thin film. It was originally developed to measure the thermal conductivity of small samples at high temperatures where the radiation heat losses were significant. Its derivatives have been further developed to determine the effective thermal conductivity of a large variety of materials including solids [30], thin films [31], wires [32], nano-ribbon [33], nanoscale junctions [34] and nanotube arrays [35], as well as composite materials [36, 37]. However, the traditional $3 \omega$ method requires the direct deposition of heater line on each sample, using microfabrication processes. These expensive microfabrications, involving extreme chemicals and environmental conditions, have limited the applications of $3 \omega$ method mostly to semiconductor materials. Recently, a reusable $3 \omega$ sensor was developed to measure the thermal conductivity of biological tissues $[38,39]$. This reusable $3 \omega$ method has great potential to be applied to measure the thermal conductivity of polymeric composites, since a lot of 
polymer material would not sustain the harsh chemical environment during the micro fabrication process in traditional $3 \omega$ method.

In this work, we investigated the enhancement of the thermal conductivity of PDMS produced by forming magnetically aligned nickel particles column/chains in the PDMS matrix. The morphology of the particle structures was characterized using optical microscope and Scanning Electron Microscope (SEM). The effective thermal conductivities of the PDMS/Ni composites were measured by a $3 \omega$ thermal conductivity measurement system. A finite element numerical modeling (FEM) was developed to predict and understand the thermal conductivity improvement of PDMS/Ni composites. The effect of the continuous column structures in PDMS matrix on thermal conductivity was thoroughly studied.

\section{Experimental and Analytical Methods}

\subsection{PDMS/Ni Composite Sample Preparation}

Nickel powders with an average diameter of $2 \mu \mathrm{m}$ and electrical resistivity of about $6.97 \mu \Omega-\mathrm{cm}$ at $20{ }^{\circ} \mathrm{C}$ were purchased from Sigma Aldrich. The Sylgard 184 Silicone elastomer kit, containing Silicone elastomer base and curing agent, was purchased from DOW CORNING Corp. The PDMS was produced by mixing the Silicone elastomer and curing agent at a weight ratio of 10 to 1 . The nickel powders were then mixed into PDMS matrix and the powder/PDMS mixture was then stirred for 5 minutes for the purpose of uniform distribution of the powders in the PDMS. After degassing the mixture in a vacuum chamber (0.254 Torr), the mixture was transferred into a mold sizing at $8 \times 8 \times 0.6 \mathrm{~mm}$ to form a typical sample. For the randomly dispersed particle composites, the sample was directly cured at $120{ }^{\circ} \mathrm{C}$ for 5 minutes to complete the cross-linking process. For the aligned particle composites, the mixture was placed between the poles of an electromagnet system (WALKER LDJ Scientific, INC. HF-7H) and the magnetic alignment process was conducted under 0.45 Tesla for 10 minutes before it was similarly cured at $120{ }^{\circ} \mathrm{C}$ within magnetic field.

\subsection{Anisotropic Thermal Conductivity Measurement based on $3 \omega$ Method}

The reusable $3 \omega$ sensor relies on a thin metal strip (usually gold), which is deposited on a glass substrate as the heat source and a thermometer. A schematic diagram of the device for PDMS/Ni composite thermal conductivity measurement is presented in The relationship between the cross-plane, thermal conductivity, $k_{y}$, and the strip theoretical temperature oscillation, $\Delta T_{t}$, is given by the following exact solution [31].

where

$$
\Delta T_{t}=\frac{-p}{\pi l k_{y}} \int_{0}^{\infty} \frac{1}{A_{1} B_{1}} \frac{\sin ^{2}(b \lambda)}{b^{2} \lambda^{2}} d \lambda
$$




$$
\begin{gathered}
B_{1}=\left(k_{x y} \lambda^{2}+\frac{i 2 \omega}{\alpha_{y}}\right)^{1 / 2} \\
k_{x y}=k_{x} / k_{y}
\end{gathered}
$$

Here, the subscripts $x$ and $y$ are used to define the directions of thermal conductivities: $x$ is parallel to the film plane (in plane) and $y$ is perpendicular to the film (cross-plane), $b$ is the heater's half-width and $\omega$ is the angular modulation frequency of the electric current, $\alpha$ is the thermal diffusivity. $p / l(\mathrm{~W} / \mathrm{m})$ represents the amplitude of the power per unit length of the stripe generated at a frequency of $2 \omega . A_{1}$ is a constant and its value depends on the boundary condition; for example, in our experiments the sample thickness is larger than the thermal penetration depth, thus the substrate can be treat as a semi-infinite body and hence, $A_{1}=-1$. For random distributed composites, the value of $k_{x y}$ is 1 , since it is totally isotropic materials. For aligned composites, the theoretically estimated value of $k_{x y}$ is from 1 to 0.75 due to the presence of isotropic glass substrate having conductivity of $0.88 \mathrm{~W} / \mathrm{mK}$. The cross-plane thermal conductivity, $k_{y}$ is obtained by . Gold (Au) strip of $19 \mathrm{~mm}$ in length, $100 \mu \mathrm{m}$ in width and $200 \mathrm{~nm}$ in thickness with four electrical connection pads was prefabricated on a glass slide using photolithography, e-beam deposition, and lift-off processes. A $5 \mathrm{~nm}$ titanium layer was deposited onto the glass surface to ensure a good adhesion between the gold deposit and the glass substrate. After the lift-off process, a $50 \mathrm{~nm}$ PMMA film was spun coated on the gold stripe to electrically insulate it from the composite samples.

\section{Figure 1}

During a typical measurement, a sample piece was placed and lightly pressed on top of the heater strip. If the glass substrate and the composite sample have thermal diffusivity ratio of 10 or less, the measured thermal conductivity could be generalized by $k_{\text {measured }}=k_{\text {glass }}+$ $k_{\text {sample }}$. Note that the higher order correlations of exact solution were neglected [38].

The operation of the device shown in Figure 1 was as follows. An AC voltage at a frequency of $1 \omega(V)$ was applied to the heater. Because of oscillatory nature of the supplied power, the temperature of the thin metal strip oscillated at a frequency of $2 \omega$. As a result of the temperature oscillation, the strip electrical resistance also oscillated at the same frequency of $2 \omega$. This resistance oscillation combined with the supplied current frequency of $1 \omega$ produced an oscillating output voltage with $3 \omega$ frequency component.

Traditionally, by monitoring the $3 \omega$ component of the output voltage of the strip, and by obtaining the linear slope of temperature gradient, $\Delta T$, vs. $\ln (\omega)$ the thermal conductivity can be determined [30]. However, this "slope method" is valid when the width of the strip is much smaller than the thermal penetration depth given by $\left(\delta=\sqrt{\alpha_{s} / 2 \omega}\right)$ of the thermal wave generated in the sample and therefore, the strip can be approximated as a line heat source. $\mathrm{A}$ detailed description of $3 \omega$ method can be found in [40]. In this research, however, the width of 
the fabricated strip was $100 \mu \mathrm{m}$ which was in the same order or larger than the thermal penetration depth of 10 to $125 \mu \mathrm{m}$, rendering "slope method" unsuitable for this measurement.

The relationship between $3 \omega$ voltage $\left(v_{3 \omega}\right)$ component over metal stripe and the strip temperature oscillation, $\Delta T$, is given by [30]

$$
\Delta T=\left[2 \frac{R_{o}}{d R / d T}\right] \frac{v_{3 \omega}}{V}
$$

where $V$ is the $1 \omega$ applied voltage; $R_{o}$ is the electrical resistance of the metal stripe; and $d R / d T$ represents the rate of change of electrical resistance with respect to temperature of the stripe, which was calibrated in vacuum within a temperature range of 300-353 K. Note that in Equation (1) the values placed in the bracket have fixed values. The $1 \omega$ voltage, $V$, is applied and the outcome $3 \omega$ voltage, $v_{3 \omega}$, is obtained by direct measurement of the non-equilibrium $3 \omega$ voltage of the Wheatstone bridge $\left(V_{3 \omega}\right)$ using a SR830 lock-in amplifier and the following equation [41]:

$$
v_{3 \omega}=\frac{R_{o}+R_{1}}{R_{1}} V_{3 \omega}
$$

The relationship between the cross-plane, thermal conductivity, $k_{y}$, and the strip theoretical temperature oscillation, $\Delta T_{t}$, is given by the following exact solution [31].

where

$$
\Delta T_{t}=\frac{-p}{\pi l k_{y}} \int_{0}^{\infty} \frac{1}{A_{1} B_{1}} \frac{\sin ^{2}(b \lambda)}{b^{2} \lambda^{2}} d \lambda
$$

$$
\begin{gathered}
B_{1}=\left(k_{x y} \lambda^{2}+\frac{i 2 \omega}{\alpha_{y}}\right)^{1 / 2} \\
k_{x y}=k_{x} / k_{y}
\end{gathered}
$$

Here, the subscripts $x$ and $y$ are used to define the directions of thermal conductivities: $x$ is parallel to the film plane (in plane) and $y$ is perpendicular to the film (cross-plane), $b$ is the heater's half-width and $\omega$ is the angular modulation frequency of the electric current, $\alpha$ is the thermal diffusivity. $p / l(\mathrm{~W} / \mathrm{m})$ represents the amplitude of the power per unit length of the stripe generated at a frequency of $2 \omega . A_{1}$ is a constant and its value depends on the boundary condition; for example, in our experiments the sample thickness is larger than the thermal penetration depth, thus the substrate can be treat as a semi-infinite body and hence, $A_{1}=-1$. For random distributed composites, the value of $k_{x y}$ is 1 , since it is totally isotropic materials. For aligned composites, the theoretically estimated value of $k_{x y}$ is from 1 to 0.75 due to the presence of isotropic glass substrate having conductivity of $0.88 \mathrm{~W} / \mathrm{mK}$. The cross-plane 
thermal conductivity, $k_{y}$ is obtained by fitting the theoretical temperature oscillation amplitude, $\Delta T_{t}$ with the measured values $\Delta T$ according to Equations (3 to 5).

\subsection{Numerical Method}

A numerical method was developed to predict and understand the anisotropic thermal conductivity of the PDMS/Ni composite materials consisting of aligned spherical particles. Figure 2 illustrates a typical unit cell and the surrounding boundary conditions. It was assumed that the heat was applied along the particle chain - the same direction as the external magnetic field.

The thickness of the particle-to-particle separation layer, filled with polymer, is an important parameter in filler-embedded composites, which profoundly affects the interfacial thermal resistance $[42,43]$. The thickness of these layers depends on magnetic strength and the restriction caused by the polymer molecular chain. The existence of the interfacial layer has been well-established, but its exact nature is not yet well understood. The thickness of the interfacial layer for PDMS/Ni composites was estimated based on the experimental works for PDMS/silica composite systems [43-44]. For example, Litvinov and Spiess [44] reported a thicknesses of $0.8 \mathrm{~nm}$ using nuclear magnetic resonance (NMR) technique whereas, Kirst et al. [45] measured thicknesses of 1 to $2.5 \mathrm{~nm}$ using dielectric spectroscopy (DRS). In this study, the average thickness of interfacial layer was assumed to be $2 \mathrm{~nm}$. In addition, the thermal properties of interfacial layer was considered to be the same as the bulk PDMS.

\section{Figure 2}

The thermal diffusion equation without volumetric heat generation is as follows:

$$
\nabla \cdot\left(k_{i} \nabla T\right)=0 \quad i=p, m
$$

The subscripts " $p$ " and " $m$ " denote the properties of particle and matrix, respectively. The total heat flow into the cell can be obtained by the integration of local heat fluxes over the bottom cross sectional area:

$$
Q=\int_{0}^{L} \int_{0}^{L}-\left.k_{m}\left(\frac{\partial T_{m}}{\partial z}\right)\right|_{z=0} d x d y
$$

Based on the heat flow obtained from computation, the effective thermal conductivity, $k_{\text {eff }}$ is determined by the following equation

$$
k_{e f f}=\frac{Q / A}{\left(T_{h w}-T_{c w}\right) / H}
$$


Where, $Q$ is the total heat flow across the bottom surface of a unit cell, $H$ is the height of the unit cell, $A$ is the cross sectional area of the unit cell, and $T_{h w}$ and $T_{c w}$ are bottom and top temperatures, respectively.

\section{Figure 3}

The numerical model was implemented on COMSOL-platform, Version 4.3, in which FEM was used to discretize the governing equation into a set of solvable algebraic equations. In these simulations, the diameter of the particles was chosen to be $2 \mu \mathrm{m}$ and the unit cell had dimensions of $10 \times 10 \times 2.002 \mu \mathrm{m}$. By varying the number of the nickel particles in the representative unit cell, starting from 1 particle and increasing it to 2, 3, and 4 particles, volume fractions of $2.09 \%, 4.18 \%, 6.27 \%$, and $8.36 \%$ were achieved, respectively. A grid independence study of the simulation model was conducted first. The effective thermal conductivity, $k_{\text {eff, }}$ results under different mesh densities are shown in .

Figure 3. As expected, the effective thermal conductivity, $k_{\text {eff, }}$ increases with increasing particle volume fraction. Also illustrated, once the FEM degree of freedoms (DOFs) reaches $100 \mathrm{~K}$, the solutions became independent of mesh size. As a result, DOFs of $200 \mathrm{k}$ was used in this work.

\section{Results \& Discussion}

\subsection{Morphology of Chain/Column Structures in PDMS}

Under an external magnetic field, dipole moments are generated in the magnetically permeable nickel particles. Two magnetic forces play major roles - one is particle-to-particle attractive force, along the magnetic field, aligning the particles in columns, and the second force acts perpendicular to the field in a repulsive mode separating columns. A stronger magnetic field will result in higher inter-particle interactions along the magnetic direction resulting in closely aligned particles while, the stronger repulsive force will separate the columns to farther distances. Borbáth et al. [46] demonstrated that when magnetic strength is increased the mean column diameter is also increased, representing a higher number of particles occupied a column. However, the number of columns per unit area decreased as the repulsive forces became stronger.

Since the column structure significantly affects the heat conduction in PDMS/Ni composite, the column morphology was quantitatively studied by varying the loading volume fraction under a fixed magnetic strength of 0.45 Tesla. The optical microscopic images of column structures of 
low volume fraction composites were obtained with Olympus Microscope (model: CX41). Figure 4 provides the optical microscopic images of random and aligned composites.

As shown in Figure 4, in the random composite, the fractal structures were randomly distributed and as it is expected, the size of these structures increases with the loading volume fraction. Without magnetic field to force particle alignment, the dimension of fractal structures augmented due to random movement of particles, (i.e., diffusion) [47]. In addition, the splitting possibility of clusters will excceed the growing probability with the increasing temperature of the composite. As a result, the bifurcation of the clusters will further increase. Fractal structures, with more branches, are believed to cause higher effective thermal conductivity in composites [48].

\section{Figure 4}

When the magnetic field was present, first the Ni particles were magnetically aligned in room temperature, and then cured. Thus, the smaller value of fractal structures were expected due to strong dipole-dipole magnetic forces, which exceeds the "naturally growth" probability of clusters [49]. The columns appeared to grow rather than split as occurred in randomly distributed Ni particle systems. As seen in Figure 4, the aligned PDMS/Ni composite manifests a better transparency in the direction of magnetic field in comparison to those of random composite due to the formation of near uniformly spaced columns.

The images of aligned composite samples (also show in Figure 4) were quantitatively analyzed using digital image processing technique by ImageJ software.

\section{Figure 5}

The average particle diameter used in this work was $2 \mu \mathrm{m}$. For magnetically aligned cases, the image processing of the columns revealed that some columns consist of a chain of single particles whereas other columns were thicker, representing two or more particles lined up in the direction of magnetic field to form the columns. Figure 5 provides the probability distribution for having single-particle chain and/or thicker columns. As seen there, most of the columns had diameters between 2 to $10 \mu \mathrm{m}$, (i.e., single to five particles), and a smaller fraction of columns had diameters larger than $10 \mu \mathrm{m}$. The diameter of some of the columns increased with increasing volumetric fraction while, the spacing between columns, (center-to-center spacing), changed insignificantly as illustrated in the middle images of Figure 4 . This may be due to the fact that the formation of column structures is mainly controlled by the dipole-dipole interaction among magnetic particles. Therefore, under a high loading situation, the short distance between magnetic particles induces a stronger repulsive force in horizontal direction, which helps form more closely packed particle column structures and as a result, the thicker columns were formed. This behavior is clearly illustrated in Figure 5 where it shows at higher 
particle loading, the probability distribution of column's size shifted to thicker values. A similar phenomena have been observed by other researchers when the magnetic field strength is increased [37], but under fixed particle loading. In their cases, stronger repulsive forces separated the columns at a greater distance reducing the total number of columns but, producing thicker columns on average. It is worth noting that, in this study, the total number of columns still had increased slightly at higher volume fractions due to the availability of particles at a constant magnetic strength.

\section{Figure 6}

The microstructure details of a single column were observed by Scanning Electron Microscope (SEM) (JEOL JSM 7401F) and optical microscope. The aligned PDMS/Ni composite samples were cut into slices along the alignment direction. The SEM image, shown in Figure 6, presents a single column microstructure. It should be noted that in the cutting process, some nickel particles could have been removed or dislocated. The presented image appears to confirm particle-to-particle proximities within nanometer ranges.

\subsection{Thermal Conductivity Measurement of Aligned and Randomly Distributed PDMS/Ni composites}

The experimental amplitude of the oscillating temperature of the strip, $\Delta T$, and the theoretical temperature amplitude, $\Delta T_{t}$, are plotted in Figure 7. It can be seen that the amplitude of the oscillatory temperature decreases at higher particle loadings indicating higher thermal conductivity. The aligned composites had lower temperature amplitudes compared to the cases for random particle distribution. The data shown in Figure 7 are used, in accordance with Equations (1-5), to obtain the composite effective thermal conductivity and the results are presented in Figure 8.

\section{Figure 7}

Figure 8 provides a comparison between numerical predictions and the experimental results of effective thermal conductivity, $k_{y}$, of composites. Representative error bars are included in Figure 8.

\section{Figure 8}

The weight scale used in this work has a 5-milligram resolution, which translates into a maximum error of about $9.85 \%$ in volume fraction evaluations. The sources of error for thermal conductivity data are concentrated mainly in the measurement of oscillating voltages and anisotropy of composites. The largest error belongs to $1 \omega$ voltage $(2 \%)$, the heater length measurements (2\%) and anisotropy of composites (7.1\%). Electrical resistance measurements 
and resistance dependency on temperature caused errors of 1 and $1.7 \%$, respectively. Other error sources are numerical integration (1\%) and $3 \omega$ voltage $(0.2 \%)$. The collective error of evaluating the effective thermal conductivity is estimated to be about $8 \%$.

The two inset plots present the isothermal line (the left inset) and the heat flux distribution along a single particle column of an aligned composites. As shown in the left inset, the nickel particle appears to be isothermal due to its large thermal conductivity relative to the surrounding matrix. Close examinations of temperature distribution near the surface of a particle, reveal that the isothermal lines are densely distributed near the surface, converging to the narrow spaces that exist between the particles. Since heat flux is proportional to temperature gradient, the lion's share of conductive heat transfer is contributed by the aligned particle column. This fact is also demonstrated by the right inset where it presents the heat flux distributions in the composite layer. As seen there, within the polymer matrix there is little, if any, heat being transferred. Whereas, pronounced heat flux lines can be observed along the particle column. The density of the heat flux lines reaches the highest value near the point contacts between particles. In the other words, the heat transfer is the strongest near these contacts.

The randomly distributed particles do not fall into a neat predictive model. However, for comparison, the Maxwell Equation (9), which was widely used for calculating thermal conductivity of a uniformly distributed particle composites, is also plotted in Figure 8 . In the numerical model, the particle conductivity, $k_{p}=90.9 \mathrm{~W} / \mathrm{mK}$, and matrix conductivity, $k_{m}=0.16$ $\mathrm{W} / \mathrm{mK}$, were considered.

$$
k_{e f f}=k_{m} \frac{2 k_{m}+k_{p}-2 \phi\left(k_{m}-k_{p}\right)}{2 k_{m}+k_{p}+\phi\left(k_{m}-k_{p}\right)}
$$

Figure 8 reveals that for the case of magnetically aligned particles, there is a good agreement between the numerical prediction and experimental measurements. However, the Maxwell Equation grossly underpredicts the thermal conductivity of particle imbedded composites. It is known that Maxwell model assumes that the isolated particles are uniformly dispersed in the matrix, which is not true for composites fabricated in this study. As described earlier, due to the particle-particle interaction, and particle aggregation fractal structures will be formed in the composites that would profoundly affect the effective thermal conductivity, especially at higher particle volume fractions.

Conductivity enhancement is strongly dependent on the fractal structures in matrix materials. Therefore, the quantitative data about the fractal structures in PDMS/Ni composite together with the interfacial thermal resistance should be used to understand the thermo-physical 
properties of composite materials. The fractal structures are mainly determined by two major mechanisms. One is the particle-particle interaction such as Van Der Waals force, magnetic force [50], gelation with short range attraction [51], and the other one is the formation of the matrix structure, such as along the grain boundary [52], and matrix interface [8]. Moreover, this study shows that the major limitation of thermal conductivity enhancement for magnetically aligned spherical particle composites is the point contacts between fillers. The non-spherical fillers such as cubic fillers could be utilized to achieve face-to-face contacts and further improve the thermal conductivity of composite materials under the magnetic alignment.

\section{Conclusion}

Magnetically aligned PDMS/Ni particle composites with varying volume fractions were prepared under an external magnetic field of 0.45 Tesla. The study of composite morphology showed that higher volume fraction results in stronger particle-particle interactions, which leads to the formation of thicker columns and closely packed particles in each column. However, in randomly distributed PDMS/Ni composites, which were processed at relatively high temperature where particle interactions were weak, the fractal clusters with large dimensions were formed. The columns formed in aligned PDMS/Ni composites provided higher thermal conductivity enhancement than that of fractal structures formed in the absence of external magnetic field. The anisotropic thermal conductivity, measured by $3 \omega$ method, showed an increase from $0.16 \mathrm{~W} / \mathrm{mK}$ to $0.725 \mathrm{~W} / \mathrm{mK}$ for aligned composites at $20 \%$ paticle volum fraction --near 4-fold enhancement. In comparison, the randomly distributed PDMS/Ni composites at $20 \%$ loading provided effective thermal conductivity of $0.422 \mathrm{~W} / \mathrm{mK}$--near half of aligned case. The SEM analysis of the composites revealed that the column thickness increased with the volumetric fractions while the spacing between the columns did not change substantially under a fixed magnetic strength. A numerical model based on column structure was built to predict the thermal conductivity enhancement and a good agreement with experimental results was achieved for aligned PDMS/Ni composites. In conclusion, these results confirmed the continuous microstructures would significantly enhance thermal conductivity of composite materials. On the other hand, thermal conductivity enhancement is limited by the point contacts between aligned spherical fillers in the composites.

\section{Acknowledgement}

The authors are grateful for the financial support received from National Science Foundation (CMMI 1348098) for this research.

\section{References}


[1] K.G. Klemic, J.F. Klemic, M.A. Reed, F.J. Sigworth, Micromolded PDMS planar electrode allows patch clamp electrical recordings from cells, Biosensors and Bioelectronics, 17(6-7) (2002) 597-604.

[2] W.-J. Chang, D. Akin, M. Sedlak, M. Ladisch, R. Bashir, Poly(dimethylsiloxane) (PDMS) and Silicon Hybrid Biochip for Bacterial Culture, Biomedical Microdevices, 5(4) (2003) 281-290.

[3] J.R. Anderson, D.T. Chiu, R.J. Jackman, O. Cherniavskaya, J.C. McDonald, H. Wu, S.H. Whitesides, G.M. Whitesides, Fabrication of Topologically Complex Three-Dimensional Microfluidic Systems in PDMS by Rapid Prototyping, Analytical Chemistry, 72(14) (2000) 3158-3164.

[4] R. Lo, P.-Y. Li, S. Saati, R. Agrawal, M.S. Humayun, E. Meng, A refillable microfabricated drug delivery device for treatment of ocular diseases, Lab on a Chip, 8(7) (2008) 1027-1030.

[5] D. Erickson, D. Sinton, D. Li, Joule heating and heat transfer in poly(dimethylsiloxane) microfluidic systems, Lab on a Chip, 3(3) (2003) 141-149.

[6] I. Sungjun, K. Banerjee, Full chip thermal analysis of planar (2-D) and vertically integrated (3-D) high performance ICs, in: Electron Devices Meeting, 2000. IEDM '00. Technical Digest. International, 2000, pp. 727-730.

[7] C. Eun Seok, K. Jae-Mo, J. Linan, R.S. Prasher, K. Min Soo, J.G. Santiago, T.W. Kenny, K.E. Goodson, Experimental study on two-phase heat transfer in microchannel heat sinks with hotspots, in: Semiconductor Thermal Measurement and Management Symposium, 2003. Ninteenth Annual IEEE, 2003, pp. 242-246.

[8] Y.P. Mamunya, V.V. Davydenko, P. Pissis, E.V. Lebedev, Electrical and thermal conductivity of polymers filled with metal powders, European Polymer Journal, 38(9) (2002) 1887-1897.

[9] G.-W. Lee, M. Park, J. Kim, J.I. Lee, H.G. Yoon, Enhanced thermal conductivity of polymer composites filled with hybrid filler, Composites Part A: Applied Science and Manufacturing, 37(5) (2006) 727-734.

[10] W. Zhou, Q. Chen, X. Sui, L. Dong, Z. Wang, Enhanced thermal conductivity and dielectric properties of Al/B-SiCW/PVDF composites, Composites Part A: Applied Science and Manufacturing, 71(0) (2015) 184-191.

[11] Y. Zhou, Y. Bai, K. Yu, Y. Kang, H. Wang, Excellent thermal conductivity and dielectric properties of polyimide composites filled with silica coated self-passivated aluminum fibers and nanoparticles, Applied Physics Letters, 102(25) (2013) 252903.

[12] K. Yang, M. Gu, Enhanced thermal conductivity of epoxy nanocomposites filled with hybrid filler system of triethylenetetramine-functionalized multi-walled carbon nanotube/silane-modified nanosized silicon carbide, Composites Part A: Applied Science and Manufacturing, 41(2) (2010) 215-221.

[13] P. Kohli, M. Sobczak, J. Bowin, M. Matthews, Advanced thermal interface materials for enhanced flip chip BGA, in: Electronic Components and Technology Conference, 2001. Proceedings., 51st, 2001, pp. 564-570.

[14] H. Dong, F. Lianhua, C.P. Wong, Effect of interface on thermal conductivity of polymer composite, in: Electronic Components and Technology Conference, 2005. Proceedings. 55th, 2005, pp. 1451-1454 Vol. 1452.

[15] R.J. Kuriger, M.K. Alam, THERMAL CONDUCTIVITY OF THERMOPLASTIC COMPOSITES WITH SUBMICROMETER CARBON FIBERS, Experimental Heat Transfer, 15(1) (2002) 19-30.

[16] L.C. Sim, S.R. Ramanan, H. Ismail, K.N. Seetharamu, T.J. Goh, Thermal characterization of Al2O3 and $\mathrm{ZnO}$ reinforced silicone rubber as thermal pads for heat dissipation purposes, Thermochimica Acta, 430(1-2) (2005) 155-165.

[17] X.Z. Niu, S.L. Peng, L.Y. Liu, W.J. Wen, P. Sheng, Characterizing and Patterning of PDMS-Based Conducting Composites, Advanced Materials, 19(18) (2007) 2682-2686.

[18] P. Yi, R.A. Awang, W.S.T. Rowe, K. Kalantar-zadeh, K. Khoshmanesh, PDMS nanocomposites for heat transfer enhancement in microfluidic platforms, Lab on a Chip, 14(17) (2014) 3419-3426.

[19] S.P. Jang, S.U.S. Choi, Role of Brownian motion in the enhanced thermal conductivity of nanofluids, Applied Physics Letters, 84(21) (2004) 4316-4318. 
[20] R. Prasher, P. Bhattacharya, P.E. Phelan, Thermal Conductivity of Nanoscale Colloidal Solutions (Nanofluids), Physical Review Letters, 94(2) (2005) 025901.

[21] T. Luo, G. Chen, Nanoscale heat transfer - from computation to experiment, Physical Chemistry Chemical Physics, 15(10) (2013) 3389-3412.

[22] H.-x. Deng, X.-I. Gong, L.-h. Wang, Development of an adaptive tuned vibration absorber with magnetorheological elastomer, Smart Materials and Structures, 15(5) (2006) N111.

[23] B. Majid, W. Xiaojie, G. Faramarz, Performance of a new magnetorheological elastomer isolation system, Smart Materials and Structures, 23(4) (2014) 045014.

[24] I. Bica, Influence of the magnetic field on the electric conductivity of magnetorheological elastomers, Journal of Industrial and Engineering Chemistry, 16(3) (2010) 359-363.

[25] J. Su, I. Mirzaee, F. Gao, X. Liu, M. Charmchi, Z. Gu, H. Sun, Magnetically Assembling Nanoscale Metal Network Into Phase Change Material-Percolation Threshold Reduction in Paraffin Using Magnetically Assembly of Nanowires, Journal of Nanotechnology in Engineering and Medicine, 5(3) (2014) 031005-031005.

[26] N. Kchit, G. Bossis, Electrical resistivity mechanism in magnetorheological elastomer, Journal of Physics D: Applied Physics, 42(10) (2009) 105505.

[27] I. Bica, The influence of hydrostatic pressure and transverse magnetic field on the electric conductivity of the magnetorheological elastomers, Journal of Industrial and Engineering Chemistry, 18(1) (2012) 483-486.

[28] X. Wang, N. Ghafoorianfar, F. Gordaninejad, Study of electrical conductivity in magnetorheological elastomers, in, 2011, pp. 797710-797710-797718.

[29] A. Boudenne, Y. Mamunya, V. Levchenko, B. Garnier, E. Lebedev, Improvement of thermal and electrical properties of Silicone-Ni composites using magnetic field, European Polymer Journal, 63(0) (2015) 11-19.

[30] D.G. Cahill, Thermal conductivity measurement from 30 to $750 \mathrm{~K}$ : the $3 \omega$ method, Review of Scientific Instruments, 61(2) (1990) 802-808.

[31] T. Borca-Tasciuc, A.R. Kumar, G. Chen, Data reduction in $3 \omega$ method for thin-film thermal conductivity determination, Review of Scientific Instruments, 72(4) (2001) 2139-2147.

[32] L. Lu, W. Yi, D.L. Zhang, $3 \omega$ method for specific heat and thermal conductivity measurements, Review of Scientific Instruments, 72(7) (2001) 2996-3003.

[33] G. Li, D. Liang, R.L.J. Qiu, X.P.A. Gao, Thermal conductivity measurement of individual Bi2Se3 nanoribbon by self-heating three- $\omega$ method, Applied Physics Letters, 102(4) (2013) 043104.

[34] R.Y. Wang, R.A. Segalman, A. Majumdar, Room temperature thermal conductance of alkanedithiol self-assembled monolayers, Applied Physics Letters, 89(17) (2006) 173113.

[35] X.J. Hu, A.A. Padilla, J. Xu, T.S. Fisher, K.E. Goodson, 3-Omega Measurements of Vertically Oriented Carbon Nanotubes on Silicon, Journal of Heat Transfer, 128(11) (2005) 1109-1113.

[36] A.R. Abramson, K. Woo Chul, S.T. Huxtable, Y. Haoquan, W. Yiying, A. Majumdar, T. Chang-Lin, Y. Peidong, Fabrication and characterization of a nanowire/polymer-based nanocomposite for a prototype thermoelectric device, Microelectromechanical Systems, Journal of, 13(3) (2004) 505-513.

[37] B.-Y. Tsui, Y. Chen-Chi, F. Kuo-Lung, Anisotropic thermal conductivity of nanoporous silica film, Electron Devices, IEEE Transactions on, 51(1) (2004) 20-27.

[38] J. Choi, S.D. Lubner, H. Natesan, Y. Hasegawa, A. Fong, C. Dames, J.C. Bischof, Thermal Conductivity Measurements of Thin Biological Tissues Using a Microfabricated 3-Omega Sensor, Journal of Medical Devices, 7(2) (2013) 020944-020944.

[39] S.D. Lubner, J. Choi, G. Wehmeyer, B. Waag, V. Mishra, H. Natesan, J.C. Bischof, C. Dames, Reusable bi-directional $3 \omega$ sensor to measure thermal conductivity of $100-\mu m$ thick biological tissues, Review of Scientific Instruments, 86(1) (2015) 014905. 
[40] B. Yang, W.L. Liu, J.L. Liu, K.L. Wang, G. Chen, Measurements of anisotropic thermoelectric properties in superlattices, Applied Physics Letters, 81(19) (2002) 3588-3590.

[41] T. Yamane, N. Nagai, S.-i. Katayama, M. Todoki, Measurement of thermal conductivity of silicon dioxide thin films using a 3w method, Journal of Applied Physics, 91(12) (2002) 9772-9776.

[42] I.V. Singh, M. Tanaka, M. Endo, Effect of interface on the thermal conductivity of carbon nanotube composites, International Journal of Thermal Sciences, 46(9) (2007) 842-847.

[43] C.-W. Nan, G. Liu, Y. Lin, M. Li, Interface effect on thermal conductivity of carbon nanotube composites, Applied Physics Letters, 85(16) (2004) 3549-3551.

[44] V.M. Litvinov, H.W. Spiess, $2 \mathrm{H}$ NMR study of molecular motions in polydimethylsiloxane and its mixtures with aerosils, Die Makromolekulare Chemie, 192(12) (1991) 3005-3019.

[45] K.U. Kirst, F. Kremer, V.M. Litvinov, Broad-band dielectric spectroscopy on the molecular dynamics of bulk and adsorbed poly(dimethylsiloxane), Macromolecules, 26(5) (1993) 975-980.

[46] T. Borbáth, S. Günther, D.Y. Borin, G. Th, S. Odenbach, X $\mu C T$ analysis of magnetic field-induced phase transitions in magnetorheological elastomers, Smart Materials and Structures, 21(10) (2012) 105018.

[47] G. Helgesen, A.T. Skjeltorp, P.M. Mors, R. Botet, R. Jullien, Aggregation of Magnetic Microspheres: Experiments and Simulations, Physical Review Letters, 61(15) (1988) 1736-1739.

[48] R. Pastor-Satorras, J.M. Rubí, Particle-cluster aggregation with dipolar interactions, Physical Review E, 51(6) (1995) 5994-6003.

[49] T.s. Vicsek, Fractal growth phenomena, World Scientific, Singapore ; Teaneck, N.J., 1989.

[50] E.S. Choi, J.S. Brooks, D.L. Eaton, M.S. Al-Haik, M.Y. Hussaini, H. Garmestani, D. Li, K. Dahmen, Enhancement of thermal and electrical properties of carbon nanotube polymer composites by magnetic field processing, Journal of Applied Physics, 94(9) (2003) 6034-6039.

[51] P.J. Lu, E. Zaccarelli, F. Ciulla, A.B. Schofield, F. Sciortino, D.A. Weitz, Gelation of particles with shortrange attraction, Nature, 453(7194) (2008) 499-503.

[52] J.W. Gao, R.T. Zheng, H. Ohtani, D.S. Zhu, G. Chen, Experimental Investigation of Heat Conduction Mechanisms in Nanofluids. Clue on Clustering, Nano Letters, 9(12) (2009) 4128-4132. 


\section{Figure Captions}

Figure 1: Schematic diagram of the thermal conductivity measurement system based on $3 \omega$ method (insets are the metal strip and a composite sample on the strip)

Figure 2: The unit cell of PDMS/Ni composites used in numerical model

Figure 3: Study of mesh size dependency on numerical outcome

Figure 4: The optical microscopic images of randomly distributed (a) and magnetically aligned (b) PDMS/Ni composites with volume fractions of 1\%, 2\%, and 3\% (The scale bars are all 200 $\mu \mathrm{m})$.

Figure 5: Effect of particle volume fraction on the column's thickness

Figure 6: The detail microscopic images of a single column in PDMS/Ni composite

Figure 7: The measured temperature rise of the $3 \omega$ strip at $300 \mathrm{~K} ;(A)$ randomly distributed composite, (B) Aligned particle composite

Figure 8: Comparison of effective thermal conductivities --measured values vs. predicted data ( insets are the isothermal line and the heat flux distribution) 


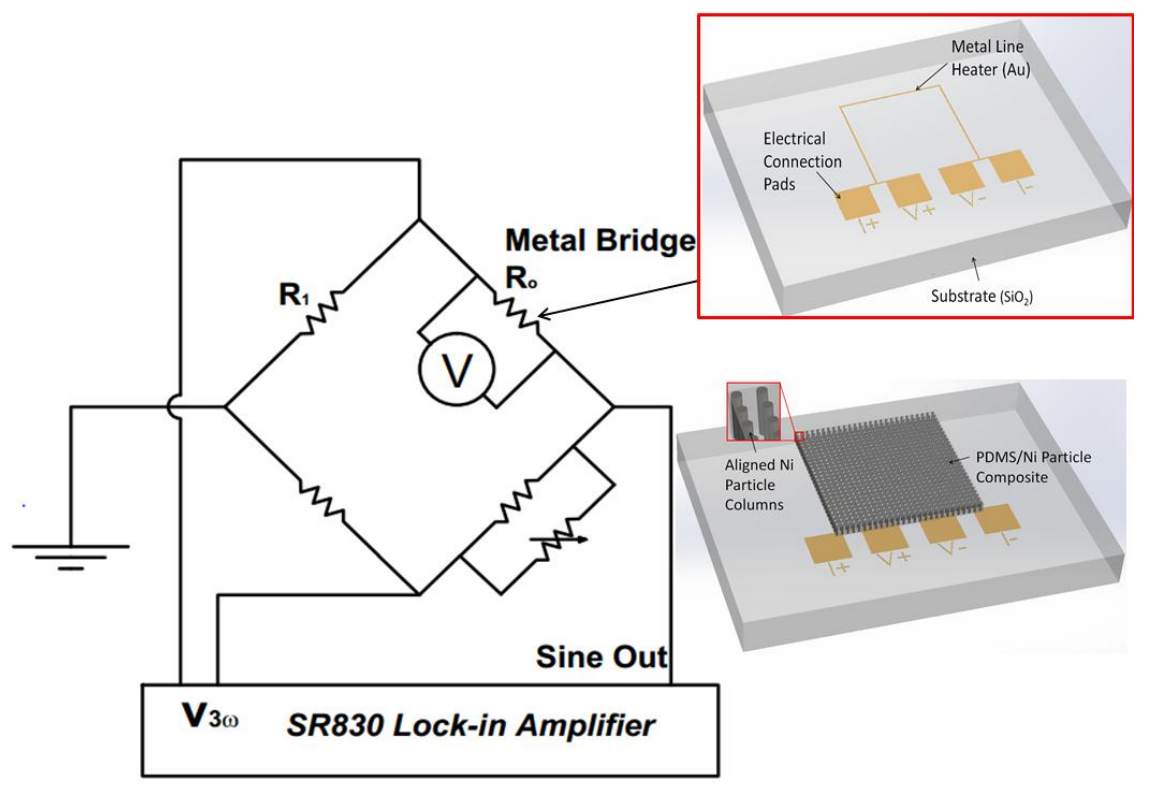

Figure 1 


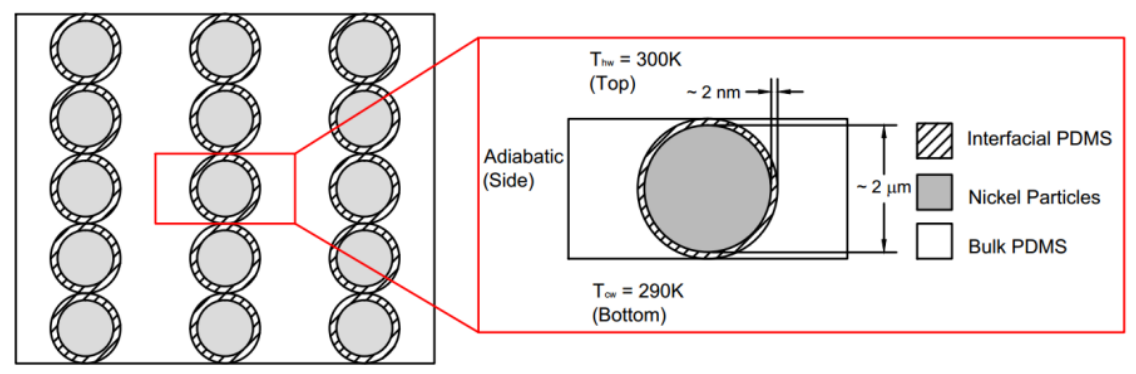

Figure 2 


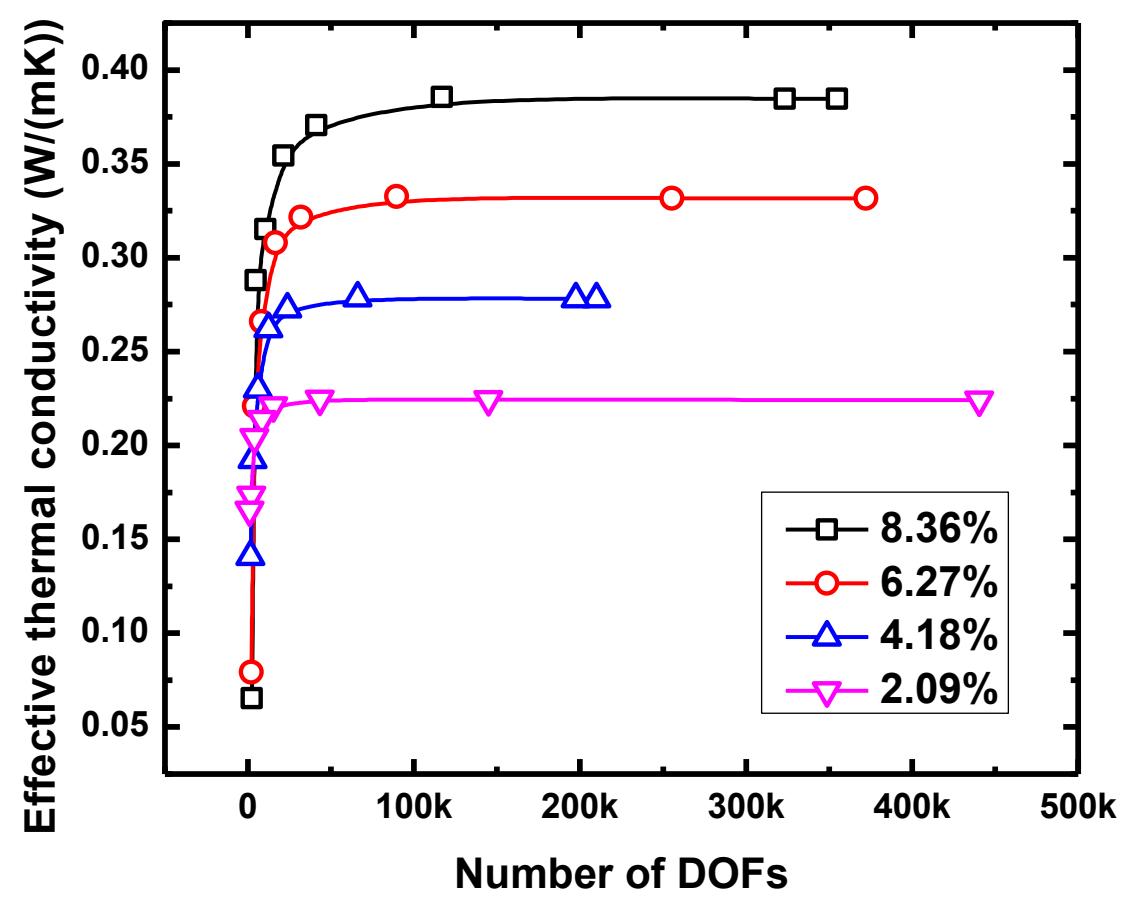

Figure 3 


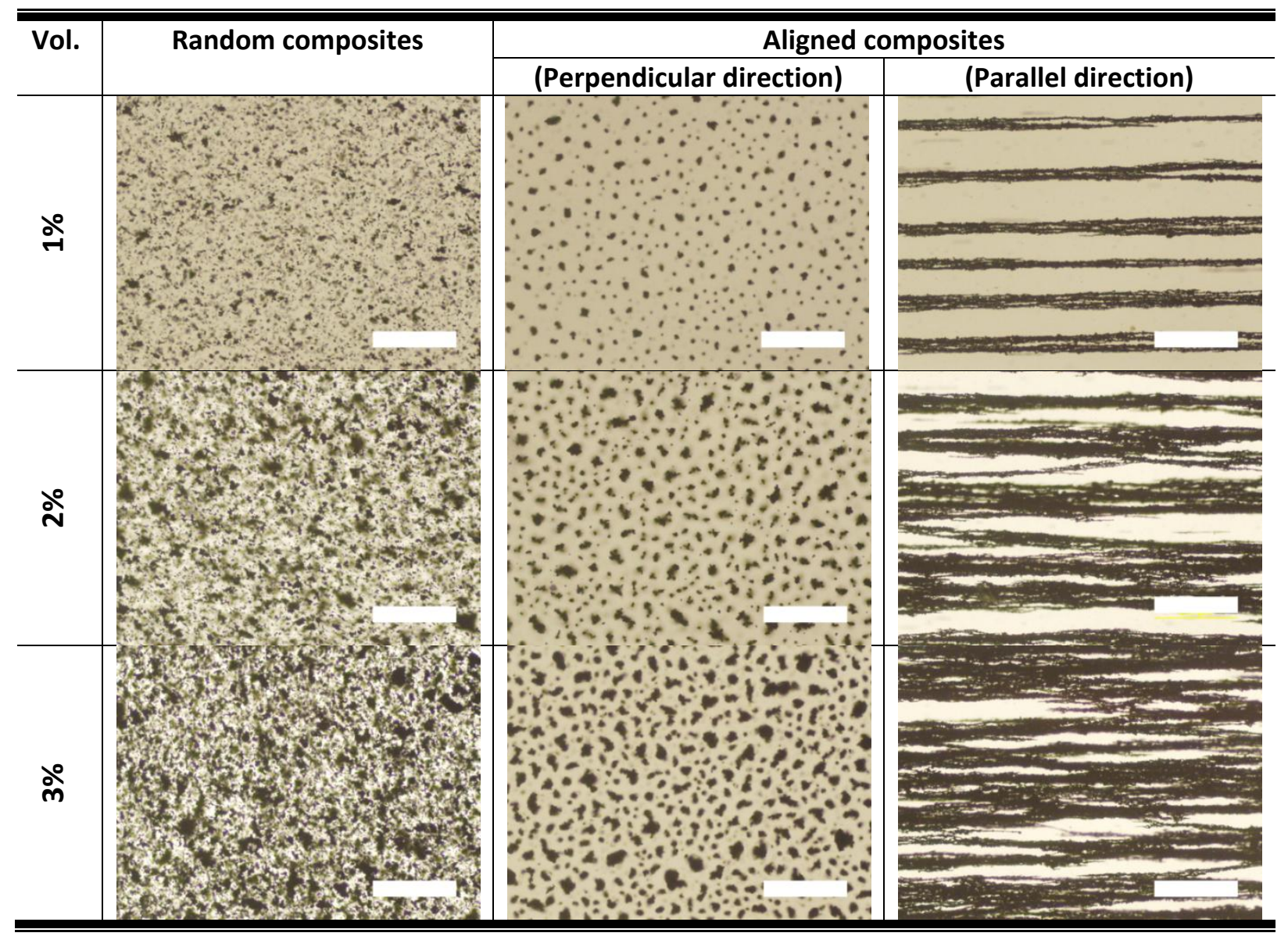

Figure 4 


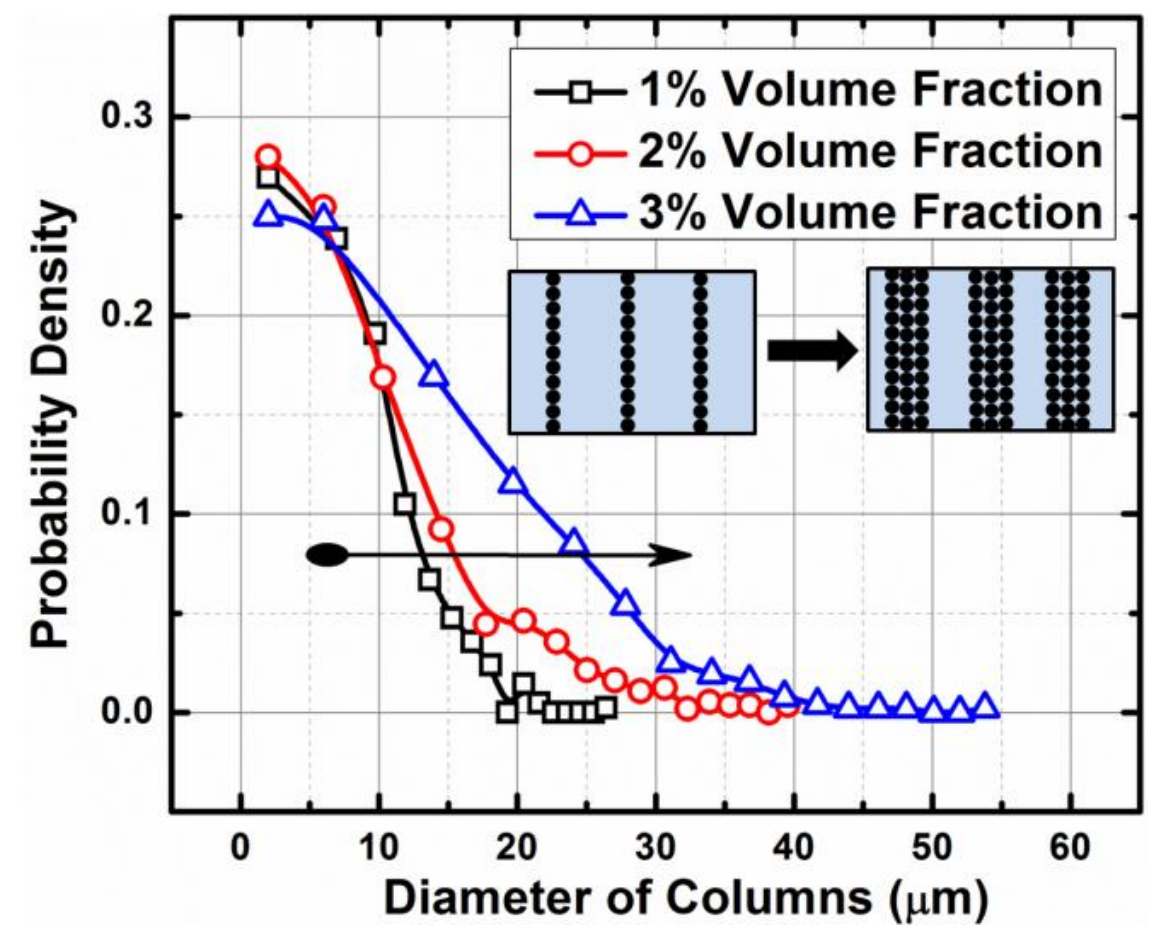

Figure 5 


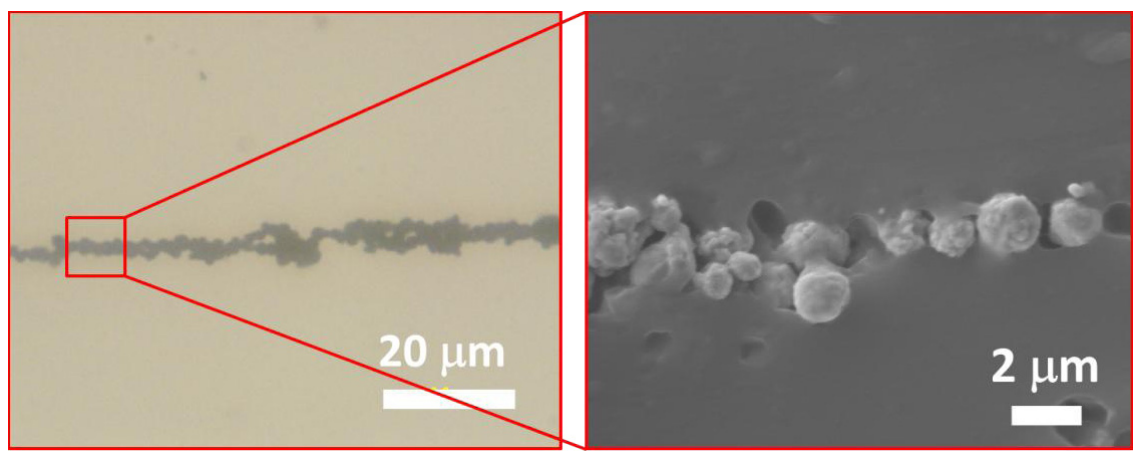

Figure 6 

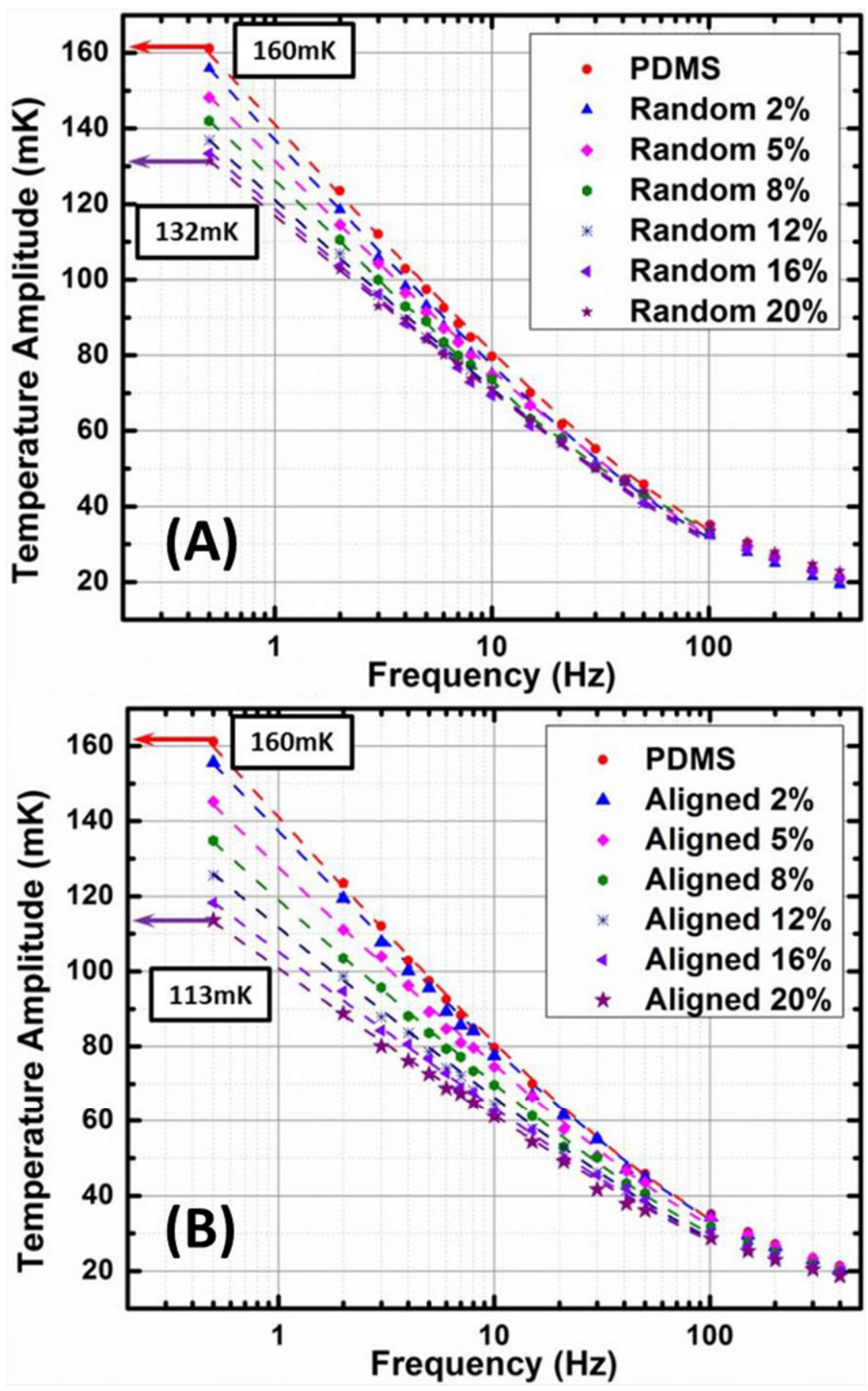

Figure 7 


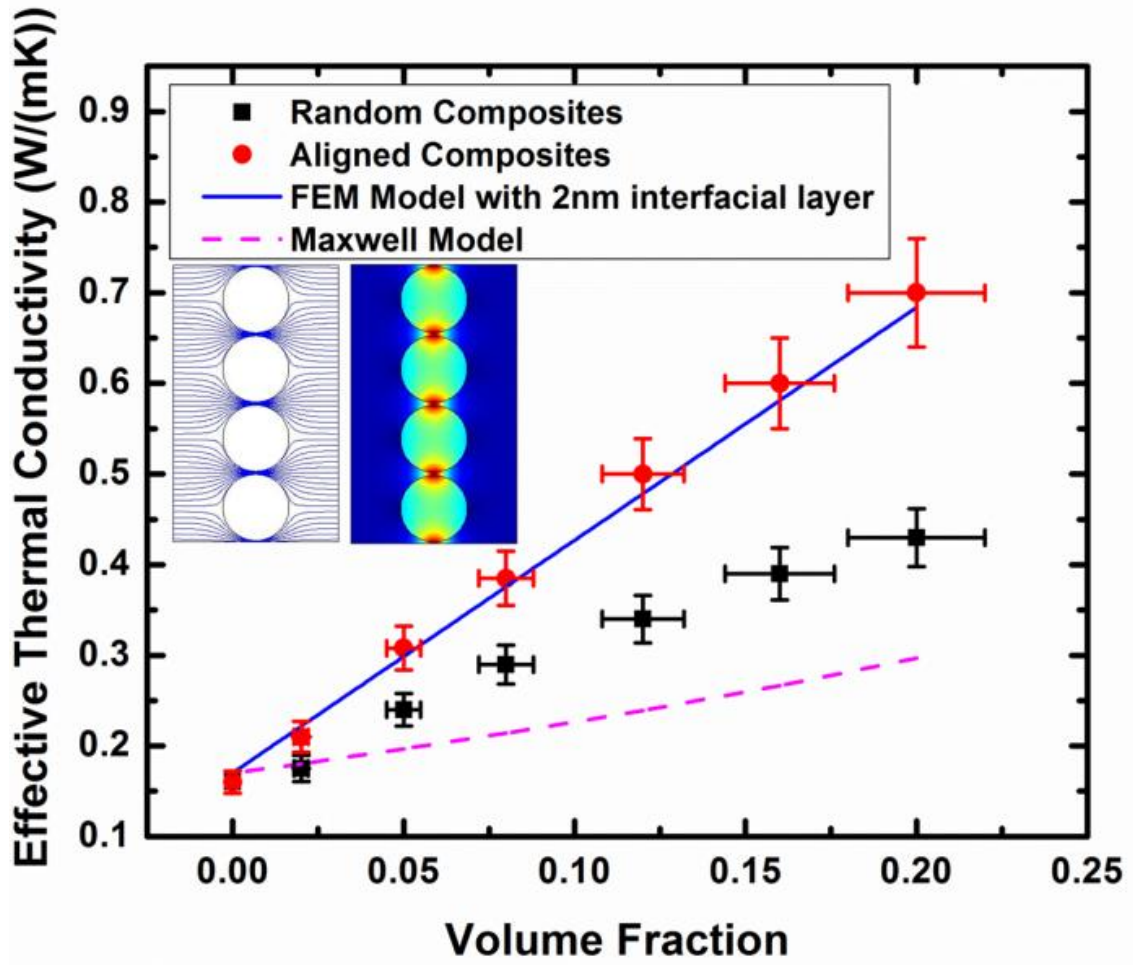

Figure 8 